\title{
Evidence for Free Precession in the Pulsar B1642-03
}

\author{
T.V. Shabanova \\ Astro Space Center of Lebedev Physical Institute, Moscow, Russia
}

J.O. Urama

Hartebeesthoek Radio Astronomy Observatory, South Africa

\begin{abstract}
The phase residuals of PSR B1642-03 obtained during a 30yr interval since 1969 to 1999 are discussed. Interpretation of observed cyclical residual changes in terms of free precession gives a wooble angle of $0.3-0.7$ degrees.
\end{abstract}

\section{Introduction}

From a timing analysis of the JPL data, Cordes \& Downs (1985) found, that the phase residuals for PSR B1642-03 have an oscillatory behavior with an amplitude of about $15 \mathrm{~ms}$. As further researches showed, PSR B1642-03 also exhibits periodic changes in the shape of the mean pulse profile with a period of about 1000 days (Cordes 1993). As Cordes suggested, cyclical timing residuals and periodic shape changes can be explain by the precession model with a wooble angle $\theta_{p} \sim 0.5^{\circ}$.

We present an analysis of the timing data of PSR B1642-03, including the archival JPL data between 1969 and 1982 (Downs \& Reichley 1983), the HartRAO timing data at $1.6 / 2.3 \mathrm{GHz}$ between 1985 and 1999 and the Pushchino timing data at $0.1 \mathrm{GHz}$ between 1991 and 1999 .

\section{The phase residuals of PSR B1642-03}

The topocentric arrival times, collected at HartRAO and PRAO observatories, were referred to the Solar System barycenter at infinite frequency using the JPL DE200 ephemeris and the astrometric parameters from Downs \& Reichley (1983). A second-order polynomial describing a secular spin-down pulsar behavior was fitted to the barycentric arrival times to obtain residuals from a timing model.

Figure 1 shows the phase residuals of PSR B1642-03 after removing the second-order polynomial from the combined JPL + HartRAO + PRAO data set of the barycentric arrival times. As can be seen, the phase residuals have an oscillatory behavior with the amplitude varying from 15 to $70 \mathrm{~ms}$ and the period varying from 3 to 7 years. A more detailed analysis of timing data showed that rotational frequency, $\nu$, and the frequency derivative, $\dot{\nu}$, are also subject to the cyclical changes. The magnitude of $\dot{\nu}$ is basically less than the mean value of 


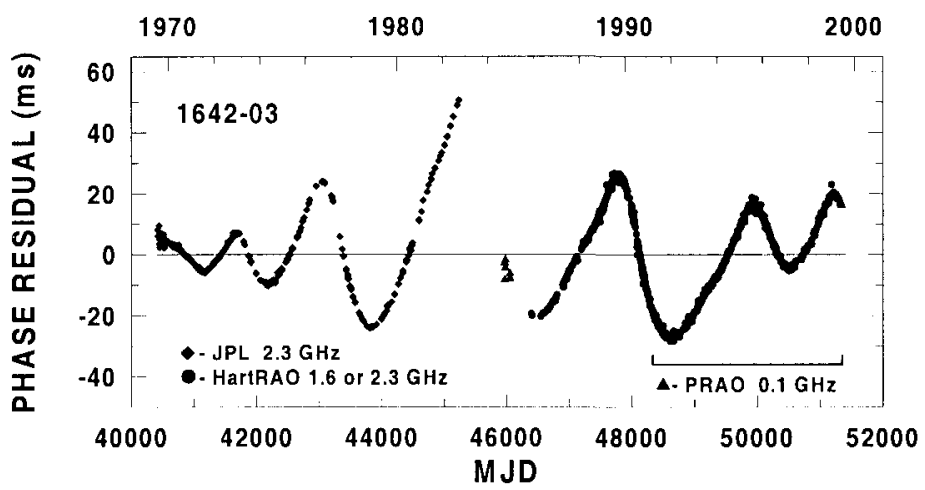

Figure 1. The phase residuals of PSR B1642-03 during a 30-yr interval of observations over $1969-1999$.

the frequency derivative and only during a short time the $\dot{\nu}$ sharply decreases by approximately $\Delta \dot{\nu} / \dot{\nu}=1.3 \%$. As a result of such spiky changes of $\dot{\nu}$ the pulsar begins to rotate $20 \mathrm{~ns}$ faster.

Simultaneous observations of PSR B1642-03 at the two widely separated frequencies of 0.1 and $1.6 / 2.3 \mathrm{GHz}$ allowed to determine the dispersion measure with improved accuracy. The new value of the DM is $35.748 \pm 0.002 \mathrm{pc} \mathrm{cm}^{-3}$. It is interesting to note, that between the two phase residual curves there is a variable time offset which shows a quasi-sinusoidal variation with a maximum amplitude of $\sim 1 \mathrm{~ms}$. Variation of this time offset, more or less, corresponds to the phase of the residual curve.

Interpretation of observed cyclical residual changes in terms of free precession gives a wooble angle between 0.3 and 0.7 degrees. If this process is really associated with free precession of the instantaneous spin axis around the symmetry axis of the neutron star crust, then the precession period can be very long (up to 7 years) and variable (from 3 to 7 years).

Acknowledgments. We are grateful to G.D. Nicolson and C.S. Flanagan for making the HartRAO pulsar data available to us. This work was supported by grant INTAS 96-0154.

\section{References}

Cordes, J. M., \& Downs, G. S. 1985, ApJS, 59, 343

Cordes, J. M. 1993, in ASP Conf. Ser. Vol. 36, Planets around Pulsars, ed. J. A. Phillips, S. E. Thorsett, \& S. R. Kulkarni (San Francisco: ASP), 43 Downs, G. S., \& Reichley, P. E. 1983, ApJS, 53, 169 